\title{
MODERATE DEVIATIONS FOR TWO SAMPLE T-STATISTICS
}

\author{
HONGYUAN CAO ${ }^{1}$
}

\begin{abstract}
Let $X_{1}, \ldots, X_{n_{1}}$ be a random sample from a population with mean $\mu_{1}$ and variance $\sigma_{1}^{2}$, and $Y_{1}, \ldots, Y_{n_{2}}$ be a random sample from another population with mean $\mu_{2}$ and variance $\sigma_{2}^{2}$ independent of $\left\{X_{i}, 1 \leq i \leq n_{1}\right\}$. Consider the two sample t-statistic $T=\frac{\bar{X}-\bar{Y}-\left(\mu_{1}-\mu_{2}\right)}{\sqrt{s_{1}^{2} / n_{1}+s_{2}^{2} / n_{2}}}$. This paper shows that $\ln P(T \geq x) \sim-x^{2} / 2$ for any $x:=x\left(n_{1}, n_{2}\right)$ satisfying $x \rightarrow \infty, x=o\left(n_{1}+n_{2}\right)^{1 / 2}$ as $n_{1}, n_{2} \rightarrow \infty$ provided $0<c_{1} \leq n_{1} / n_{2} \leq c_{2}<\infty$. If, in addition, $E\left|X_{1}\right|^{3}<\infty, E\left|Y_{1}\right|^{3}<\infty$, then $\frac{P(T \geq x)}{1-\Phi(x)} \rightarrow 1$ holds uniformly in $x \in\left(0, o\left(\left(n_{1}+n_{2}\right)^{1 / 6}\right)\right)$.
\end{abstract}

Mathematics Subject Classification. 60F10, 60G50, 62F05.

Received July 14, 2006. Revised December 12, 2006.

\section{INTRODUCTION AND MAIN RESULTS}

Let $\left\{X, X_{n}, n \geq 1\right\}$ be a sequence of independent non-degenerate real-valued random variables on the probability space $(\Omega, \Sigma, P)$. Put

$$
S_{n}=\sum_{i=1}^{n} X_{i}, \quad B_{n}^{2}=\sum_{i=1}^{n} E X_{i}^{2}, \quad V_{n}^{2}=\sum_{i=1}^{n} X_{i}^{2} .
$$

In classical limit theorems, moment conditions and other related conditions are sufficient and usually necessary (see Petrov 1995 [3]). For instance, for independent and identically distributed (i.i.d) random variables, the central limit theorem holds if and only if $E X^{2} I(|X| \leq x)$ is slowly varying as $x \rightarrow \infty$. On the other hand, limit theorems for self-normalized sums $S_{n} / V_{n}$ put those classical limit theorems on a new perspective. Shao (1997) [5] showed that no moment conditions are needed for the self-normalized large deviation result

$$
\frac{S_{n}}{V_{n} \sqrt{n}}
$$

and that the tail probability of $S_{n} / V_{n}$ is Gaussian like when $\mathrm{X}$ is in the domain of attraction of the normal law and sub-Gaussian like when $\mathrm{X}$ is in the domain of attraction of a stable law. Giné et al. (1997) [1] proved that the tails of $S_{n} / V_{n}$ are uniformly sub-Gaussian when the sequence is stochastically bounded whereas Shao

Keywords and phrases. Two sample t-statistic, asymptotic distribution, moderate deviation.

1 Department of Statistics and Operations Research, University of North Carolina-Chapel Hill, Chapel Hill, NC 27599, USA; hycao@email.unc.edu

(C) EDP Sciences, SMAI 2007 
(1999) [6] found that a Cramér type result for self-normalized sums holds only under a finite third moment condition. What's more, some finer results were obtained on the self-normalized limit theorems for independent but not necessarily identically distributed random variables. Jing et al. (2003) [2] obtained a Cramér-type large deviation result for general independent random variables with zero means and finite variances. They showed that

$$
P\left(S_{n} / V_{n} \geq x\right)=(1-\Phi(x))\left(1+O(1)(1+x)^{3} B_{n}^{-3} \sum_{i=1}^{n} E\left|X_{i}\right|^{3}\right)
$$

for $0 \leq x \leq B_{n} /\left(\sum_{i=1}^{n} E\left|X_{i}\right|^{3}\right)^{1 / 3}$, where $O(1)$ is bounded by an absolute constant. Some refinements of this result may be found in [4]. The above result is useful because it not only supplies the relative error but also a Berry-Esseen rate of convergence. Besides, Jing, Shao and Wang proved that the exponential moment condition needed for the normalized sum can be remarkably reduced to only the finite moment condition of low order, and hence such a large deviation have applications to a variety of fields, and in particular, to statistics. From the statistics point of view, self-normalization is more fit to do inferences because the parameters involved in many classical limit theorems are usually unknown, one has to use some statistics to estimate them first and then apply the estimators in the classical limit theorems. This commonly used practice is indeed the self-normalization.

The main purpose of this paper is to study the moderate deviation for the two-sample t-statistics. We will show that the main result of Jing et al. (2003) [2] holds for the two-sample t-statistics.

Let $X_{1}, \ldots, X_{n_{1}}$ be a sample of i.i.d. random variables with mean $\mu_{1}$ and variance $\sigma_{1}^{2}$ and $Y_{1}, \ldots, Y_{n_{2}}$ be another sample of i.i.d. random variables with mean $\mu_{2}$ and variance $\sigma_{2}^{2}$, independent of $X_{1}, \ldots, X_{n_{1}}$. Consider the two sample t-statistic

where

$$
T=\frac{\bar{X}-\bar{Y}-\left(\mu_{1}-\mu_{2}\right)}{\sqrt{s_{1}^{2} / n_{1}+s_{2}^{2} / n_{2}}}
$$

$$
\begin{gathered}
\bar{X}=\sum_{i=1}^{n_{1}} X_{i}, \quad \bar{Y}=\sum_{i=1}^{n_{2}} Y_{i}, \\
s_{1}^{2}=\frac{1}{n_{1}-1} \sum_{i=1}^{n_{1}}\left(X_{i}-\bar{X}\right)^{2}, s_{2}^{2}=\frac{1}{n_{2}-1} \sum_{i=1}^{n_{2}}\left(Y_{i}-\bar{Y}\right)^{2} .
\end{gathered}
$$

This statistic is frequently used to construct confidence interval and do hypothesis testing for the difference between two means. There are several premises underlying the use of two sample t-test. It is assumed that the data has been derived from populations with normal distribution. Based on the fact that $s_{i} \rightarrow \sigma_{i}$ a.s. for $i=1,2$, with moderate violation of the assumption, quite often statisticians recommend to use the two sample t-test provided the samples are not too small and the samples are of equal or nearly equal size.

The aim of this paper is to give a theoretical justification for the use of two-sample t-statistics when the populations are not normally distributed. The main results are moderate deviations for the two-sample t-statistic.

Theorem 1.1. Assume that there are $0<c_{1} \leq c_{2}<\infty$ such that

$$
c_{1} \leq n_{1} / n_{2} \leq c_{2}
$$

Then for any $x:=x\left(n_{1}, n_{2}\right)$ satisfying $x \rightarrow \infty, x=o\left(\left(n_{1}+n_{2}\right)^{1 / 2}\right)$

$$
\ln P(T \geq x) \sim-x^{2} / 2
$$

as $n_{1}, n_{2} \rightarrow \infty$, where $a_{n} \sim b_{n}$ means $\lim _{n \rightarrow \infty} a_{n} / b_{n}=1$.

Theorem 1.2. Let $n=n_{1}+n_{2}$. Assume that $E\left|X_{1}\right|^{3}<\infty, E\left|Y_{1}\right|^{3}<\infty$ and (1.2) is satisfied. Then

$$
\frac{P(T \geq x)}{1-\Phi(x)}=1+O(1)(1+x)^{3} n^{-1 / 2} d^{3}
$$


for $0 \leq x \leq n^{1 / 6} / d$, where $d^{3}=\left(E\left|X_{1}-\mu_{1}\right|^{3}+E\left|Y_{1}-\mu_{2}\right|^{3}\right) /\left(\sigma_{1}^{2}+\sigma_{2}^{2}\right)^{3 / 2}$ and $O(1)$ is a finite constant depending only on $c_{1}$ and $c_{2}$. In particular, we have

$$
\frac{P(T \geq x)}{1-\Phi(x)} \rightarrow 1
$$

uniformly in $x \in\left(0, o\left(n^{1 / 6}\right)\right)$.

It is well-known that when $\sigma_{1}=\sigma_{2}$, one can use the pooled two-sample t-statistic $T^{*}$ defined by

$$
T^{*}=\frac{\bar{X}-\bar{Y}-\left(\mu_{1}-\mu_{2}\right)}{\sqrt{s^{* 2}\left(1 / n_{1}+1 / n_{2}\right)}}
$$

where

$$
s^{* 2}=\frac{\left(n_{1}-1\right) s_{1}^{2}+\left(n_{2}-1\right) s_{2}^{2}}{n_{1}+n_{2}-2} .
$$

We have similar results for $T^{*}$.

Theorem 1.3. Assume that (1.2) is satisfied. Then for any $x:=x\left(n_{1}, n_{2}\right)$ satisfying $x \rightarrow \infty, x=o\left(\left(n_{1}+\right.\right.$ $\left.n_{2}\right)^{1 / 2}$ )

$$
\ln P\left(T^{*} \geq x\right) \sim-x^{2} / 2
$$

as $n_{1}, n_{2} \rightarrow \infty$.

Theorem 1.4. Assume $E\left|X_{1}\right|^{3}<\infty$ and $E\left|Y_{1}\right|^{3}<\infty$. Assume also (1.2) is satisfied. Then

$$
\frac{P\left(T^{*} \geq x\right)}{1-\Phi(x)}=1+O(1)(1+x)^{3} n^{-1 / 2} d^{3}
$$

for $0 \leq x \leq n^{1 / 6} / d$, where $d^{3}=\left(E\left|X_{1}-\mu_{1}\right|^{3}+E\left|Y_{1}-\mu_{2}\right|^{3}\right) /\left(\sigma_{1}^{2}+\sigma_{2}^{2}\right)^{3 / 2}$ and $O(1)$ is a finite constant depending only on $c_{1}$ and $c_{2}$. In particular, we have

$$
\frac{P\left(T^{*} \geq x\right)}{1-\Phi(x)} \rightarrow 1
$$

uniformly in $x \in\left(0, o\left(n^{1 / 6}\right)\right)$.

Remark 1.1. We remark that it is not necessary to assume finite variances of $X_{1}$ and $Y_{1}$ in Theorem 1.1. Theorem 1.1 remains valid when both $X_{1}$ and $Y_{1}$ are in the domain of attraction of a normal law. See the proof of Theorem 1.1.

\section{Proofs}

Our proofs are based on self-normalized large and moderate deviations for independent random variables. We restate them below for easy reference.

Proposition 2.1 [5], Th. 3.1, Rem. 4.2). Assume that $\left\{X_{n}, n \geq 1\right\}$ is a sequence of i.i.d. random variables with $E\left(X_{1}\right)=0$ and $E\left(X_{1}^{2}\right)<\infty$. Then there exist $0<\varepsilon_{0} \leq 1$ and $n_{0}$ such that for any $1 / \varepsilon_{0} \leq x \leq \varepsilon_{0} \sqrt{n}$ and $n \geq n_{0}$

$$
P\left(S_{n} \geq x V_{n}\right) \leq \exp \left(-x^{2} / 4\right) .
$$

Next proposition is an extension of Theorem 3.1 of Shao (1997) [5]. 
Proposition 2.2. Let $\left\{X_{i}, 1 \leq i \leq n_{1}\right\}$ and $\left\{Y_{i}, 1 \leq i \leq n_{2}\right\}$ be two independent sequences of i.i.d. random variables with zero means. Let $a_{n, 1}$ and $a_{n, 2}$ be two sequences of real numbers. Assume that the following conditions are satisfied:

(i) both $X_{1}$ and $Y_{1}$ are in the domain of attraction of a normal law;

(ii) $a_{n, 1} \rightarrow a_{1}$ and $a_{n, 2} \rightarrow a_{2}$ as $n \rightarrow \infty$, where $a_{1} \neq 0, a_{2} \neq 0$;

(iii) $n_{1} / n \rightarrow b_{1}$ and $n_{2} / n \rightarrow b_{2}$ as $n \rightarrow \infty$, where $0<b_{1}<\infty, 0<b_{2}<\infty$.

Then for any sequence of positive numbers $\left\{x_{n}, n \geq 1\right\}$ with $x_{n} \rightarrow \infty$ and $x_{n}=o(\sqrt{n})$ as $n \rightarrow \infty$

$$
\lim _{n \rightarrow \infty} x_{n}^{-2} \ln P\left(\frac{a_{n, 1} \sum_{i=1}^{n_{1}} X_{i}+a_{n, 2} \sum_{i=1}^{n_{2}} Y_{i}}{\left(a_{n, 1}^{2} \sum_{i=1}^{n_{1}} X_{i}^{2}+a_{n, 2}^{2} \sum_{i=1}^{n_{2}} Y_{i}^{2}\right)^{1 / 2}} \geq x_{n}\right)=-\frac{1}{2} .
$$

Proof. The proof follows the same lines of that of Theorem 3.1 in [5] except that we define $l(x)$ and $z_{n}$ in (4.3) in [5] as follows

and

$$
l(x)=a_{1}^{2} b_{1} E\left(X_{1}^{2} I\left\{\left|X_{1}\right| \leq x\right\}\right)+a_{2}^{2} b_{2} E\left(Y_{1}^{2} I\left\{\left|Y_{1}\right| \leq x\right\}\right)
$$

$$
z_{n}=\inf \left\{s: s>b+1, \frac{l(s)}{s^{2}} \leq \frac{x_{n}^{2}}{n}\right\}, \text { where } b=\inf \{x \geq 1: l(x)>0\} .
$$

The details are omitted here.

Let $\left\{X_{i}, i \geq 1\right\}$ be independent random variables. Assume $E X_{i}=0$ and $0<E X_{i}^{2}<\infty$. For convenience, we introduce

$$
\Delta_{n, x}=\frac{(1+x)^{2}}{B_{n}^{2}} \sum_{i=1}^{n} E X_{i}^{2} I_{\left\{\left|X_{i}\right|>B_{n} /(1+x)\right\}}+\frac{(1+x)^{3}}{B_{n}^{3}} \sum_{i=1}^{n} E\left|X_{i}\right|^{3} I_{\left\{\left|X_{i}\right| \leq B_{n} /(1+x)\right\}}
$$

for $x \geq 0$.

Proposition 2.3 [2], Th. 2.1). There is an absolute constant $A(>1)$ such that

$$
\frac{P\left(S_{n} \geq x V_{n}\right)}{1-\Phi(x)}=\mathrm{e}^{O(1) \Delta_{n, x}} \quad \text { and } \quad \frac{P\left(S_{n} \leq-x V_{n}\right)}{\Phi(-x)}=\mathrm{e}^{O(1) \Delta_{n, x}}
$$

for all $x \geq 0$ satisfying

and

$$
x^{2} \max _{1 \leq i \leq n} E X_{i}^{2} \leq B_{n}^{2}
$$

where $|O(1)| \leq A$.

$$
\Delta_{n, x} \leq(1+x)^{2} / A,
$$

Proposition 2.4 [2], Th. 2.3). Let $0<\delta \leq 1$ and set

$$
L_{n, \delta}=\sum_{i=1}^{n} E\left|X_{i}\right|^{2+\delta}, d_{n, \delta}=B_{n} / L_{n, \delta}^{1 /(2+\delta)} .
$$

Then

$$
\frac{P\left(S_{n} / V_{n} \geq x\right)}{1-\Phi(x)}=1+O(1)\left(\frac{1+x}{d_{n, \delta}}\right)^{2+\delta}
$$

and

$$
\frac{P\left(S_{n} / V_{n} \leq-x\right)}{\Phi(-x)}=1+O(1)\left(\frac{1+x}{d_{n, \delta}}\right)^{2+\delta}
$$


for $0 \leq x \leq d_{n, \delta}$, where $O(1)$ is bounded by an absolute constant. In particular, if $d_{n, \delta} \rightarrow \infty$ as $n \rightarrow \infty$, we have

$$
\frac{P\left(S_{n} \geq x V_{n}\right)}{1-\Phi(x)} \rightarrow 1, \quad \frac{P\left(S_{n} \leq-x V_{n}\right)}{\Phi(-x)} \rightarrow 1
$$

uniformly in $0 \leq x \leq o\left(d_{n, \delta}\right)$.

The main idea of the proofs is to first get rid of $\bar{X}$ and $\bar{Y}$ in the denominator of $T$ and then to apply the moderate deviations, Propositions 2.3 and 2.4. Without loss of generality, we assume that $\mu_{1}=\mu_{2}=0$. If we can prove theorems under 0 means, let $X^{\prime}=X-\mu_{1}, Y^{\prime}=Y-\mu_{2}$, the results hold for $T^{\prime}=\frac{X^{\prime}-Y^{\prime}}{\sqrt{s_{1}^{\prime 2} / n_{1}+s_{2}^{\prime 2} / n_{2}}}$. Yet here, $s_{1}^{\prime 2}=s_{1}^{2}, s_{2}^{\prime 2}=s_{2}^{2}$ and $T=T^{\prime}$. So the result is true for $T$. Further, we define

$$
V_{1}^{2}=\sum_{i=1}^{n_{1}} X_{i}^{2}, \quad V_{2}^{2}=\sum_{i=1}^{n_{2}} Y_{i}^{2}
$$

We prove Theorem 1.1 first.

Proof of Theorem 1.1. The first step is to get rid of $\bar{X}$ and $\bar{Y}$ in the denominator of $T$. Notice that

$$
\begin{aligned}
s_{1}^{2} & =\frac{1}{n_{1}-1} \sum_{i=1}^{n_{1}}\left(X_{i}-\bar{X}\right)^{2} \\
& =\frac{1}{n_{1}-1}\left(\sum_{i=1}^{n_{1}} X_{i}^{2}-n_{1} \bar{X}^{2}\right) \\
& =\frac{1}{n_{1}-1} V_{1}^{2}\left(1-\frac{n_{1} \bar{X}^{2}}{V_{1}^{2}}\right)
\end{aligned}
$$

and similarly,

$$
s_{2}^{2}=\frac{1}{n_{2}-1} V_{2}^{2}\left(1-\frac{n_{2} \bar{Y}^{2}}{V_{2}^{2}}\right)
$$

Let

$$
\bar{s}_{1}^{2}=\frac{V_{1}^{2}}{n_{1}-1}, \quad \bar{s}_{2}^{2}=\frac{V_{2}^{2}}{n_{2}-1} .
$$

Set

$$
\bar{T}=\frac{\bar{X}-\bar{Y}}{\sqrt{\bar{s}_{1}^{2} / n_{1}+\bar{s}_{2}^{2} / n_{2}}} .
$$

Then we can see for any $x \geq 0$ and $0<\varepsilon<1 / 2$

$$
P(\bar{T} \geq x) \leq P(T \geq x) \leq P(\bar{T} \geq x \sqrt{1-\varepsilon})+P\left(\frac{n_{1} \bar{X}^{2}}{V_{1}^{2}} \geq \varepsilon\right)+P\left(\frac{n_{2} \bar{Y}^{2}}{V_{2}^{2}} \geq \varepsilon\right)
$$

From Proposition 2.1, we can get for $1 /\left(\varepsilon_{0}^{2} \min \left(n_{1}, n_{2}\right)\right) \leq \varepsilon \leq \varepsilon_{0}^{2}$

$$
P\left(\frac{n_{1} \bar{X}^{2}}{V_{1}^{2}} \geq \varepsilon\right) \leq 2 \exp \left(-\varepsilon n_{1} / 4\right)
$$


and

$$
P\left(\frac{n_{2} \bar{Y}^{2}}{V_{2}^{2}} \geq \varepsilon\right) \leq 2 \exp \left(-\varepsilon n_{2} / 4\right)
$$

with $n_{1}$ and $n_{2}$ large enough.

To apply Proposition 2.3, we first verify that conditions (2.3) and (2.4) are satisfied. Because of (1.2), without loss of generality, assume

$$
n_{1}=b_{1} n, n_{2}=b_{2} n, \quad b_{1}+b_{2}=1 \text { with } b_{1}>0 \text { and } b_{2}>0
$$

In our case, we introduce a new sequence of independent random variables $\left\{Z_{i}\right\}$ defined as below:

$$
Z_{i}= \begin{cases}X_{i} / n_{1} & \text { for } 1 \leq i \leq n_{1} \\ -Y_{i-n_{1}} / n_{2} & \text { for } n_{1}<i \leq n\end{cases}
$$

Then

$$
\begin{gathered}
E Z_{i}^{2}=\frac{1}{n^{2}}\left\{\begin{array}{l}
\sigma_{1}^{2} / b_{1}^{2} \text { for } 1 \leq i \leq n_{1} \\
\sigma_{2}^{2} / b_{2}^{2} \text { for } n_{1}<i \leq n
\end{array} \approx \frac{1}{n^{2}},\right. \\
B_{n}^{2}=\sum_{i=1}^{n} E\left|Z_{i}\right|^{2}=\frac{1}{n}\left(\sigma_{1}^{2} / b_{1}^{2}+\sigma_{2}^{2} / b_{2}^{2}\right) \approx \frac{1}{n},
\end{gathered}
$$

where $a \approx b$ means $0<\liminf a / b \leq \limsup a / b<\infty$. So as long as $x=o\left(\left(n_{1}+n_{2}\right)^{1 / 2}\right)$, condition (2.3) is satisfied.

Next, we turn to condition (2.4). Since $n_{1} Z_{i}, 1 \leq i \leq n_{1}$ are independent having the same distribution as $X_{1}$, and $n_{2} Z_{i}, n_{1}<i \leq n$ are independent having the same distribution as $Y_{1}$, we have

$$
\begin{aligned}
\Delta_{n, x}= & \frac{(1+x)^{2}}{B_{n}^{2}} \sum_{i=1}^{n} E Z_{i}^{2} I_{\left\{\left|Z_{i}\right|>B_{n} /(1+x)\right\}}+\frac{(1+x)^{3}}{B_{n}^{3}} \sum_{i=1}^{n} E\left|Z_{i}\right|^{3} I_{\left\{\left|Z_{i}\right| \leq B_{n} /(1+x)\right\}} \\
\leq & \frac{(1+x)^{2} E X_{1}^{2} I_{\left\{\left|X_{1}\right|>\sigma_{1} \sqrt{n_{1}} /(1+x)\right\}}}{E X_{1}^{2}}+\frac{(1+x)^{3} E\left|X_{1}\right|^{3} I_{\left\{\left|X_{1}\right| \leq \sqrt{n_{1}} \sqrt{\sigma_{1}^{2}+c_{2} \sigma_{2}^{2}} /(1+x)\right\}}}{n_{1}^{1 / 2}\left(E X_{1}^{2}\right)^{3 / 2}} \\
& +\frac{(1+x)^{2} E Y_{1}^{2} I_{\left\{\left|Y_{1}\right|>\sigma_{2} \sqrt{n_{2}} /(1+x)\right\}}}{E Y_{1}^{2}}+\frac{(1+x)^{3} E\left|Y_{1}\right|^{3} I_{\left\{\left|Y_{1}\right| \leq \sqrt{n_{2}} \sqrt{\sigma_{2}^{2}+\sigma_{1}^{2} / c_{1}} /(1+x)\right\}}}{n_{2}^{1 / 2}\left(E Y_{1}^{2}\right)^{3 / 2}} \\
= & o\left((1+x)^{2}\right)
\end{aligned}
$$

by the fact that $x=o\left(\left(n_{1}+n_{2}\right)^{1 / 2}\right)$ and that $E \xi^{2} I_{\{|\xi|>t\}}=o(1)$ and $E|\xi|^{3} I_{\{|\xi| \leq t\}}=o(t)$ as $t \rightarrow \infty$ for any random variable $\xi$ with a finite second moment.

This proves that conditions (2.3) and (2.4) are satisfied. Hence by Proposition 2.3

$$
\ln P(\bar{T} \geq x) \sim-x^{2} / 2
$$

for $x \rightarrow \infty$ and $x=o\left(\left(n_{1}+n_{2}\right)^{1 / 2}\right)$. Combining (2.8), (2.9), (2.10) and (2.14) yields (1.3) by the arbitrariness of $\varepsilon$.

Proof of Theorem 1.2. From Proposition 2.4, we get

$$
\frac{P(\bar{T} \geq x)}{1-\Phi(x)}=1+O(1)(1+x)^{3} n^{-1 / 2} d^{3}
$$


and

$$
\frac{P(\bar{T} \geq x \sqrt{1-\varepsilon})}{1-\Phi(x \sqrt{1-\varepsilon})}=1+O(1)(1+x)^{3} n^{-1 / 2} d^{3}
$$

for $0 \leq x \leq n^{1 / 6} / d$ and $0<\varepsilon \leq 1 / 2$. Choose $\varepsilon(x, n)$ properly, say, $\varepsilon(x, n)=(1+x) / \sqrt{n}$.

Noting that when $x \rightarrow \infty$,

we have

$$
1-\Phi(x) \sim \frac{\mathrm{e}^{-x^{2} / 2}}{\sqrt{2 \pi} x}
$$

$$
\begin{aligned}
\frac{1-\Phi(x \sqrt{1-\varepsilon})}{1-\Phi(x)} & =1+\frac{\Phi(x)-\Phi(x \sqrt{1-\varepsilon})}{1-\Phi(x)} \\
& =1+O(1) \frac{\left(\mathrm{e}^{-x^{2}(1-\varepsilon) / 2}-\mathrm{e}^{-x^{2} / 2}\right) / x}{\mathrm{e}^{-x^{2} / 2} / x} \\
& =1+O(1)\left(\mathrm{e}^{\varepsilon x^{2} / 2}-1\right) \\
& =1+O\left(\varepsilon x^{2}\right)=1+O(1)(1+x)^{3} n^{-1 / 2} .
\end{aligned}
$$

For other $x$,

$$
\begin{aligned}
\frac{1-\Phi(x \sqrt{1-\varepsilon})}{1-\Phi(x)} & =1+\frac{\Phi(x)-\Phi(x \sqrt{1-\varepsilon})}{1-\Phi(x)} \\
& =1+O(1) \int_{x \sqrt{1-\varepsilon}}^{x} \frac{1}{\sqrt{2 \pi}} \mathrm{e}^{-t^{2} / 2} \mathrm{~d} t \\
& =1+O(1) \int_{x \sqrt{1-\varepsilon}}^{x} \frac{t}{x \sqrt{1-\varepsilon} \sqrt{2 \pi}} \mathrm{e}^{-t^{2} / 2} \mathrm{~d} t \\
& =1+O(1)\left(\mathrm{e}^{\varepsilon x^{2} / 2}-1\right) \\
& =1+O\left(\varepsilon x^{2}\right)=1+O(1)(1+x)^{3} n^{-1 / 2}
\end{aligned}
$$

because $\varepsilon x^{2}=O(1)$ and $\varepsilon=O(1)(1+x) n^{-1 / 2}$. Also note that by Proposition 2.1,

$$
\begin{aligned}
P\left(n_{1} \bar{X}^{2} / V_{1}^{2} \geq \varepsilon\right) & =P\left(\frac{\left(\sum_{i=1}^{n_{1}} X_{i}\right)^{2}}{\sum_{i=1}^{n_{1}} X_{i}^{2}} \geq \varepsilon n_{1}\right) \\
& \leq \exp \left(-\varepsilon n_{1} / 4\right)=\exp \left(-\varepsilon b_{1} n / 4\right) \\
& =\exp \left(-\varepsilon b_{1} n / 8\right) \exp \left(-\varepsilon b_{1} n / 8\right) \\
& =o\left(n^{-1}\right)(1-\Phi(x))
\end{aligned}
$$

by noting that $\log n=o(\varepsilon n)$ and $x^{2}=o(\varepsilon n)$. Similarly, we have

$$
P\left(n_{2} \bar{Y}^{2} / V_{2}^{2} \geq \varepsilon\right)=o\left(n^{-1}\right)(1-\Phi(x))
$$

thus,

$$
\begin{aligned}
& P\left(n_{1} \bar{X}^{2} / V_{1}^{2} \geq \varepsilon\right)=o\left((1+x)^{3} n^{-1 / 2}(1-\Phi(x))\right), \\
& P\left(n_{2} \bar{Y}^{2} / V_{2}^{2} \geq \varepsilon\right)=o\left((1+x)^{3} n^{-1 / 2}(1-\Phi(x))\right)
\end{aligned}
$$


and

$$
1-\Phi(x \sqrt{1-\varepsilon})=(1-\Phi(x))\left(1+O(1)(1+x)^{3} n^{-1 / 2}\right)
$$

hold, then taking into account of (2.8), the result of Theorem 1.2 follows.

The proofs of Theorems 1.3 and 1.4 are along the same line as that of Theorems 1.1 and 1.2 , so the details are omitted here.

Acknowledgements. I would like to thank Professor Z.Y. Lin for his advice and guidance throughout my work with this paper.

\section{REFERENCES}

[1] E. Giné, F. Gőtze, and D.M. Mason, When is the student t-statistic asymptotically standard normal? Ann. Probab. 25 (1997) 1514-1531.

[2] B.-Y. Jing, Q.M. Shao, and Q.Y. Wang, Self-normalized Cramér-type large deviations for independent random variables. Ann. Probab. 31 (2003) 2167-2215.

[3] V.V. Petrov, Limit Theorems of Probability Theory. Clarendon Press, Oxford (1995).

[4] J. Robinson, and Q. Wang, On the self-normalized Cramér-type large deviation. J. Theor. Probab. 18 (2005) $891-909$.

[5] Q.M. Shao, Self-normalized large deviations. Ann. Probab. 25 (1997) 285-328.

[6] Q.M. Shao, A Cramér type large deviation result for student's t-statistic. J. Theor. Probab. 12 (1999) 385-398. 